\title{
Multiple analysis of mitochondrial metabolism, autophagy and cell
}

\section{death}

\author{
LIU $D^{1}$., LAI H.T ${ }^{1}$, PEYRE $F^{1}$., BRENNER $\mathrm{C}^{1}$
}

1 Université Paris-Saclay, Institut Gustave Roussy, CNRS, Aspects métaboliques et systémiques de l'oncogénèse pour de nouvelles approches thérapeutiques, 94805, Villejuif, France

Corresponding author: Dr C. Brenner

METSY - UMR 9018 CNRS

Université Paris-Saclay

Gustave Roussy, PR2

114 rue Edouard Vaillant

94805 Villejuif, France

Tel: 33-1 42115128

E-mail: catherine.brenner@universite-paris-saclay.fr

Key words: autophagy, cell death, cytoxicity, metabolism, mitochondria, viability 


\section{Table of Contents}

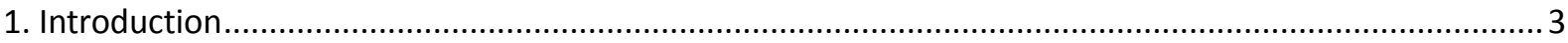

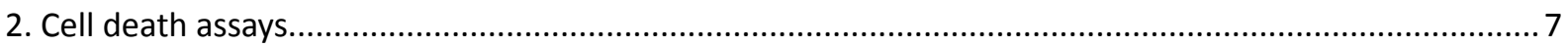

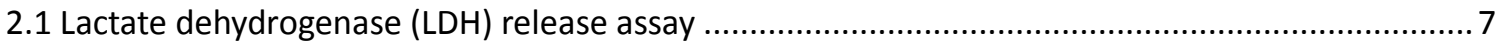

2.1.1 Materials, reagents and equipment ................................................................. 7

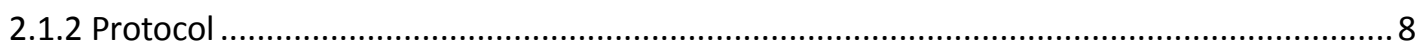

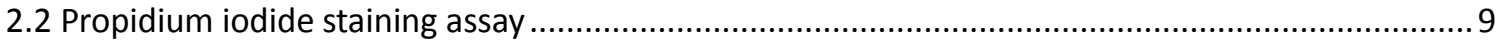

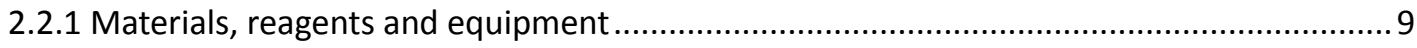

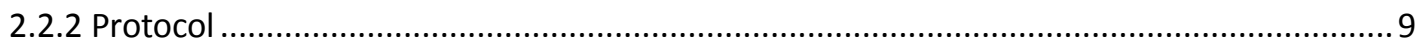

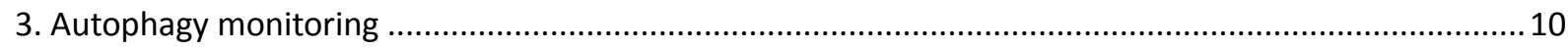

3.1 Autophagic biomarker evaluation by western-blot............................................................... 10

3.1.1 Materials, reagents and equipment, ................................................................ 10

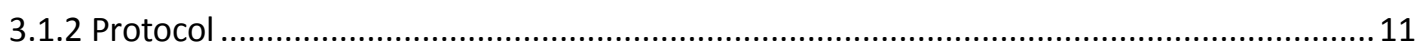

3.2 Autophagosome formation using GFP-LC3 plasmid transfection.............................................. 13

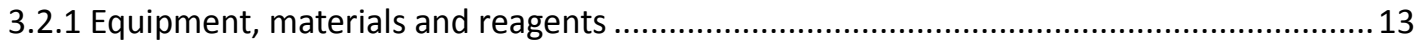

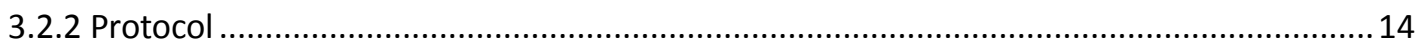

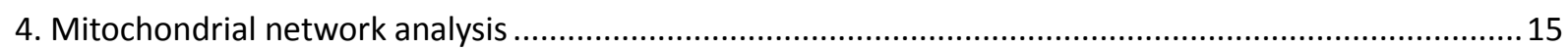

4.1 Equipment, materials and reagents .................................................................. 15

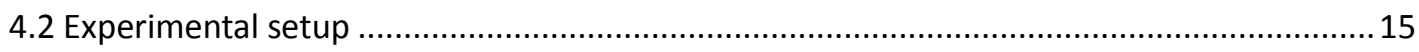

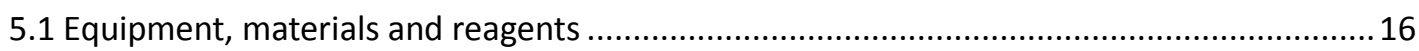

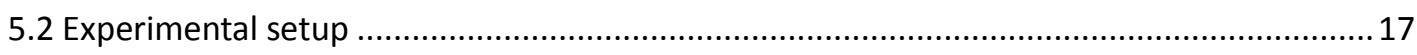

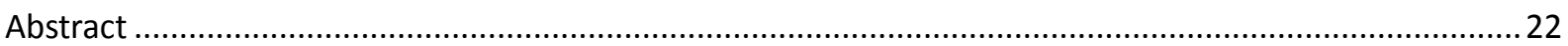

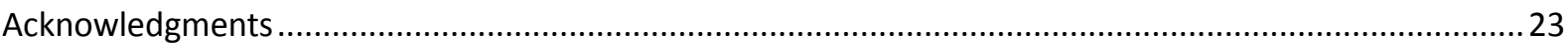

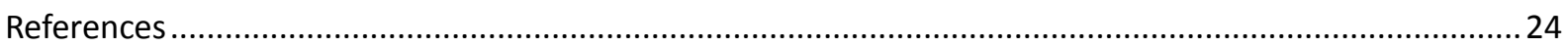

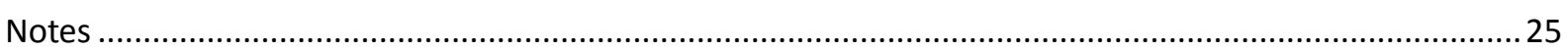

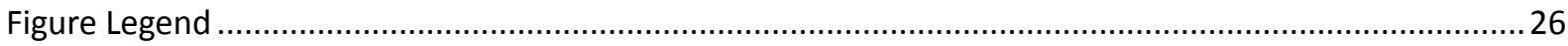




\section{Introduction}

For a long time, cardiomyocytes are considered as pertinent cell models in toxicology [1, 2]. In fact, many drugs have deleterious side effects on cardiomyocytes, triggering acute tissue damage and cardiac dysfunction. As a result, cardiomyopathy can develop in surviving treated patients affecting their quality of life or even their survival.

In the heart, cardiomyocytes constitute the beating cells consuming important amount. Unfortunately, they have limited energy reserve capacity and are highly dependent on mitochondrial energy production for contraction. Moreover, at the adult stage, these cells stop proliferating and have limited repair capacity. Altogether, these properties render cardiomyocytes particularly vulnerable to metabolism perturbation and cellular injury by exogenous stimuli. As a prototypic example among many clinically used drugs, doxorubicin, an anti-cancer agent having strong anti-tumoral activity, has cumulative dose-dependent cardiotoxic effects. Indeed, doxorubicin can induce apoptosis of cardiomyocytes mediated, at least in part, by oxidative stress, mitochondrial dysfunction and metabolic failure [1, 2]. Therefore, a better understanding of cardiomyocyte stress response and the development of new innocuous drugs for cardiac cells are urgently needed $[1,2]$.

To identify new compounds that preserve cardiomyocyte viability, it is mandatory to monitor simultaneously their cellular effects on cell death and also their mode of action on various intracellular signaling pathways. Here, we proposed joint robust protocols to analyze cellular cardiotoxicity of compounds and determine whether their cellular impact involves (1) cell death, (2) autophagy, (3) effect on mitochondria network and/or (4) energetic metabolism. 
Cell death modalities are classified according to morphological, biochemical and functional criteria and can be routinely divided into accidental cell death or programmed cell death relying on a complex molecular machinery [3]. Thus, necrosis represents the prototypic accidental cell death characterized by early plasma membrane permeabilization, increase in cell volume and release of internal constituents in the extracellular environment. On the opposite, apoptosis refers to programmed cell death involving sequential signaling events such as caspase activation, nuclear degradation and late plasma membrane permeabilization with the exposure of specific lipids to the cell surface (i.e., phosphatidylserine) and leakage of cytosolic constituents.

In normal heart, the level of cell death is extremely low and in response to heart injury, apoptosis and necrosis can both occur in vivo. However, in cell culture, there is a clear kinetic distinction between apoptosis versus necrosis, plasma membrane integrity being lost within minutes $(<1 \mathrm{~h})$ in necrosis whereas only late in apoptosis $(>24 \mathrm{~h})[3]$. As a consequence, the duration of cell treatment can be exploited to determine whether cell death occur by apoptosis versus necrosis.

In our assayas, plasma membrane permeabilisation is reflected by the quantification of lactate dehydrogenase (LDH) activity released in the extracellular medium. Indeed, LDH is a stable cytosolic enzyme that is released upon cell lysis, with a half-life of approximately $9 \mathrm{~h}$. It catalyzes the interconversion of pyruvate and lactate with concomitant interconversion of $\mathrm{NADH}$ and NAD+, allowing the evaluation of cell death using tetrazolium salts in conjunction with diaphorase [4-5]. Alternatively, cell death can also be measured by the entry of a 
non-permeant fluorescent compound into nucleus of live cells, such as propidium iodide (PI) and detected by spectrofluorimetry.

Autophagy is an evolutionally-conserved process, dedicated to the elimination of damaged cell organelles such as mitochondria or denaturated/aggregated proteins [6]. Generally, autophagy is mostly known as a cell survival mechanism. However, in some circumstances, autophagy can be also a letal processes in connection with apoptosis and other cell death pathways or not [3].Upon initiation phase of autophagy, Autophagy related 12 protein (Atg12) conjugates with autophagy related 5 (Atg5) to promote the recruitment and conversion of microtubule-associated proteins light chain 1A/1B and light chain $3 \mathrm{~B}$ (LC3-I) to the membrane-bound LC3-phosphatidylethanolamine conjugate (LC3-II) forming autophagic vacuoles [7]. LC3-II and these vacuoles, namely autophagosomes, can be used as specific marker to monitor autophagy by western-blot or fluorescence microscopy respectively.

Mitochondria are adaptable organelles playing an essential role in energetic metabolism for ATP production, intermediate metabolite production, redox state and cell fate [8-10]. Mitochondria population forms a dynamic network, which can be fused or fragmented in response to many intra- and extracellular stress signals. Thus, changes in the morphology of the mitochondrial network can be induced by the availability of energy source (e.g. pyruvate, glutamine, fatty acids), reactive oxygen species (ROS), pharmacological agents, as well as by radiations. The balance between fusion and fission is linked to mitochondrial biogenesis or autophagic removal of dysfunctional mitochondria, thereby contributing to maintain a pool of functional mitochondria. Mitochondria can also participate to cell death trough the 
permeabilization of their membrane, an irreversible process triggered by the release of mitochondrial proteins into the cytosol such as cytochrome $c$, apoptosis inducing factor (AIF) and endonuclease G (Endo G) [3,11]. This process called mitochondrial membrane permeabilization (MMP) is usually accompanied by mitochondrial membrane potential loss $(\Delta \Psi \mathrm{m})$, ROS production and ATP fall, namely a metabolic catastrophe [3, 11]. A mode of action of compounds involving effects on mitochondria can be analyzed by fluorescence confocal microscopy and 3D reconstruction using specific fluorescent probes.

Here, we describe complementary cell biology methods that can be combined for a better efficacy at limited cost. These miniaturized and reproducible assays are developed for neonatal rat ventricular cardiomyocytes (RNVCs) and immortalized cardiomyoblasts (H9c2 cells) in microtiter plates, allowing robotization for high throughput if needed. For pedagogical purpose, examples of results obtained with a set of control molecules are shown. Hydrogen peroxide $\left(\mathrm{H}_{2} \mathrm{O}_{2}\right)$ is used as a classical cardiac cell death inducer. Rapamycin is used as an inducer of autophagy and Digoxin and Digitoxigenin, two cardiac glycosides used to treat heart failure in clinics, are used for their ability to protect cardiomyocytes from oxidative stress induced cell death. 


\section{Cell death assays}

Here, two kind of assays are described. The first assay, namely Lactate deshydrogenase (LDH) release assay, offers the advantage to analyze the supernatant of culture cells following a particular treatment, whereas the second assay, propidium iodide (PI) assay, labels directly the nucleus in live cells, i.e. without any fixation. Please refer to note 1 and 2.

\subsection{Lactate dehydrogenase (LDH) release assay}

\subsubsection{Materials, reagents and equipment}

1) Isolated neonatal rat ventricular cardiomyocytes (RNVCs) [12]

2) CytoTox $96 \AA$ non-radioactive cytotoxicity assay kit (stored at $\left.-20^{\circ} \mathrm{C}\right)$, (Promega, G1780). One assay kit contains sufficient substrate mix, assay buffer and stop solution for 1,000 cell-mediated cytotoxicity assays in 96-well plate format, includes 5 vials of substrate mix, $60 \mathrm{ml}$ assay buffer, $25 \mu \mathrm{LDH}$ positive control, $5 \mathrm{ml}$ lysis solution (10X), $65 \mathrm{ml}$ stop solution

3) DMEM (Dulbecco's Modified Eagle Medium), high glucose, GlutaMAX ${ }^{\mathrm{TM}}$ Supplement, $\left(\mathrm{Gibco}^{\mathrm{TM}}, 10566016\right)$

4) 96-Well Microtiter Microplates, (Thermo Scientific ${ }^{\mathrm{TM}}, 2205$ )

5) Digoxin (Sigma-Aldrich, D6003). Digitoxigenin (Sigma-Aldrich, D9404)

6) Tecan Infinite 200 spectrofluorimeter (Männedorf, Switzerland) or similar spectrofluorimeter equipped with a microplate reader able to record absorbance at 490nm. 


\subsubsection{Protocol}

\subsubsection{Reagent preparation for CytoTox 96® assay kit}

1) Thaw Assay Buffer, remove $12 \mathrm{ml}$ and promptly store the unused portion at $-20^{\circ} \mathrm{C}$

2) Warm $12 \mathrm{ml}$ of Assay Buffer to room temperature; keep protected from light

3) Add $12 \mathrm{ml}$ of room-temperature Assay Buffer to a plastic bottle of Substrate Mix to form the CytoTox 96® Reagent

4) Invert and shake gently to dissolve the substrate

\subsubsection{Assay}

1) Seed RNVC into 96 -well plate $\left(40,000\right.$ cells per well in $100 \mu 1$ plating medium at $\left.37^{\circ} \mathrm{C}\right)[14]$

2) Remove medium and treat cardiomyocytes by $100 \mu \mathrm{l}$ of $10 \mu \mathrm{M}$ Digitoxigenin, $10 \mu \mathrm{M}$ Digoxin, $0.1 \%$ DMSO (negative control) and lysis buffer (positive control) for $2 \mathrm{~h}$ at $37^{\circ} \mathrm{C}$

3) After $2 \mathrm{~h}$, replace all the treatments by $100 \mu \mathrm{l}$ of $200 \mu \mathrm{M} \mathrm{H}_{2} \mathrm{O}_{2} / \mathrm{lysis}$ buffer

4) $2 \mathrm{~h}$ later, transfer $25 \mu \mathrm{l}$ supernatant from the cell culture plate to a new plate. At this step, continue the protocol or store the supernatant containing plate at $-80^{\circ} \mathrm{C}$ until use

5) Add $25 \mu \mathrm{l}$ of the CytoTox $96 \AA$ Reagent to each sample aliquot

6) Cover the plate with foil or an opaque box to protect it from light and incubate for 30min at room temperature

7) Add $25 \mu 1$ of Stop Solution to each well of the 96-well plate

8) Pop any large bubbles using a syringe needle before recording the absorbance at 490nm with Tecan Infinite 200 spectrofluorimeter within $1 \mathrm{~h}$ after adding the stop solution 
9) Analyze the data using one-way ANOVA, Sidak's multiple comparisons test to determine statistical significance of results comparing cell treatment to vehicle treatment (i.e. DMSO)

10) Figure 1 shows an example of results.

\subsection{Propidium iodide staining assay}

\subsubsection{Materials, reagents and equipment}

1) Propidium Iodide (PI, Invitrogen, $\mathrm{P} 3566), 1 \mathrm{mM}$ stock solution in $\mathrm{PBS}, \mathrm{pH} 7.4$, $\left(\mathrm{Gibco}^{\mathrm{TM}}\right.$ 10010031)

2) DMEM (Dulbecco's Modified Eagle Medium), high glucose, GlutaMAX ${ }^{\mathrm{TM}}$ Supplement, $\left(\mathrm{Gibco}^{\mathrm{TM}}, 10566016\right)$

3) Lysis buffer: $25 \mathrm{mM}$ Tris, $\mathrm{HCl} \quad(\mathrm{pH} \quad 7.6) \quad 150 \mathrm{mM} \quad \mathrm{NaCl}, 1 \% \mathrm{NP}-40, \quad 1 \%$ sodium deoxycholate, $0.1 \%$ SDS)

4) 96 Well-TC-treated Microplates (flat bottom) (Corning®, CLS3596)

5) Tecan Infinite 200 spectrofluorimeter (Männedorf, Switzerland) or similar spectrofluorimeter equipped with a microplate reader to record fluorescence at $\lambda$ ex: $530 \mathrm{~nm}$ and $\lambda$ em: $620 \mathrm{~nm}$

\subsubsection{Protocol}

1) Seed RNVC into 96 -well plate $(40,000$ cells per well in $100 \mu 1$ plating medium)

2) Remove medium and treat cells were treated by $100 \mu 1$ of $10 \mu \mathrm{M}$ Digitoxigenin, $10 \mu \mathrm{M}$

Digoxin, $0.1 \%$ DMSO (negative control) and lysis buffer (positive control) for $2 \mathrm{~h}$ 
3) After $2 \mathrm{~h}$, replace all treatments by $100 \mu \mathrm{l}$ of $200 \mu \mathrm{M} \mathrm{H}_{2} \mathrm{O}_{2}$ or lysis buffer

4) After $2 \mathrm{~h}$, add $1 \mu \mathrm{l}$ of $100 \mu \mathrm{M}$ PI in culture medium

5) Read fluorescence with Tecan Infinite 200 spectrofluorimeter ( $\lambda$ ex: $530 \mathrm{~nm} ; \lambda$ em: $620 \mathrm{~nm}$ )

6) Analyze the data using one-way ANOVA, Sidak's multiple comparisons test

7) Figure 2 shows an example of results.

\section{Autophagy monitoring}

Here, we describe two independent protocols to detect autophagy biomarker by the analysis of LC3I conversion to LC3II by western-blot and the counting of autophagosome based on the detection of gfp-LC3 by fluorescence microscopy.

\subsection{Autophagic biomarker evaluation by western-blot}

\subsubsection{Materials, reagents and equipment,}

\subsubsection{Materials and reagents}

1) LC3B (D11) antibody (Cell Signaling Technology, 3868)

2) $\beta$-Actin (C4) antibody (Santa Cruz Biotechnology, sc-47778)

3) Lysis buffer: 20mM Hepes, 150mM NaCl, 2mM EDTA, 10\% Glycerol, 0.50\% NP-40, $1 \mu \mathrm{M}$ microcystin. For $5 \mathrm{ml}$ buffer, add half pill of mini EDTA-free protease inhibitor tablets (complete ${ }^{\text {TM }}$ Protease Inhibitor Cocktail, Roche, 11697498001)

4) Loading buffer (5x): $300 \mathrm{mM}$ Tris, $50 \%$ glycerol, $12.5 \%$ dodecylsulfate (SDS), $50 \mathrm{mM}$ dithiothreitol (DTT), $0.05 \%$ bromophenol blue, $\mathrm{pH} 6.8$

5) $4-15 \%$ precast gel (Bio-Rad Mini-PROTEAN TGX) 
6) Migration buffer (10x): weigh $30 \mathrm{~g}$ Tris base, $144 \mathrm{~g}$ glycine, $10 \mathrm{~g}$ SDS. Add water to a volume of $950 \mathrm{ml}$. Mix and adjust $\mathrm{pH}$ to 8.3. Complete to $1 \mathrm{~L}$ with water

7) DTT (10x): weigh $1.54 \mathrm{~g}$ DTT and make up to $10 \mathrm{ml}$ with water. Aliquot and store at $-20{ }^{\circ} \mathrm{C}$ or use fresh

8) Trans-Blot Turbo Mini $0.2 \mu \mathrm{m}$ PVDF Transfer Packs, (Bio-Rad, 1704156)

9) Phosphate-buffered saline (PBS)-Tween (10x): weigh $624 \mathrm{mg}$ sodium dihydrogen phosphate monohydrate $\left(\mathrm{NaH}_{2} \mathrm{PO}_{4}\right) 2.98 \mathrm{~g} \mathrm{Na}_{2} \mathrm{HPO}_{4}$ - sodium phosphate dibasic $\left(\mathrm{Na}_{2} \mathrm{HPO}_{4}\right)$, $204.5 \mathrm{~g}$ sodium chloride $(\mathrm{NaCl}), 12.5 \mathrm{ml}$ Tween 20 , and make up to $2.5 \mathrm{~L}$ with water. Pre-warm PBS for cell washes at $37^{\circ} \mathrm{C}$

10) Ultrasensitive enhanced chemiluminescent (ECL) substrate, e.g., Supersignal West Femto Maximum Sensitivity Substrate, (Thermo Scientific, 4094)

11) Bicinchoninic acid (BCA) kit (Sigma)

12) Rapamycin (Sigma)

13) 6 Well TC-treated Microplates (Corning®, flat bottom, CLS3506)

\subsubsection{Equipment}

1) Trans-blot Turbo transfer system, Bio-Rad

2) Gel imaging system, e.g., Chemidoc (Bio-Rad)

3) Image $\mathbf{J}$ software (NIH, open source)

\subsubsection{Protocol}

4) Seed $2 \times 10^{6}$ of RNVC per well in 6-well cell culture plate in $3 \mathrm{ml}$ of plating medium/well

5) Treat cells by $3 \mathrm{ml}$ of $1 \mu \mathrm{M}$ Digitoxigenin, $1 \mu \mathrm{M}$ Digoxin, $3 \mu \mathrm{M}$ rapamycin (positive 
control) and $0.01 \%$ DMSO (negative control) for $6 \mathrm{~h}$

6) Aspirate the culture medium and wash the wells with warm PBS for two times, then add $200 \mu \mathrm{l}$ of lysis buffer / well

7) Use the dispenser to detach the cells with lysis buffer

8) Collect all the cells and lysis buffer and transfer them into microtubes, keep tubes on ice for 30min

9) Centrifuge at $12,000 \mathrm{~g}$ for $10 \mathrm{~min}$ at $4{ }^{\circ} \mathrm{C}$

10) Transfer the supernatant into new microtubes and put them on ice

11) Determine protein concentration in an aliquot by the BCA method following the manufacturer's instruction using bovine serum albumin as standard.

12) Add $20 \mu \mathrm{g}$ of samples, $4 \mu \mathrm{l}$ of loading buffer $(5 \times), 2 \mu \mathrm{l}$ of DTT (10x) into a microtube, (total volume is $20 \mu \mathrm{l}$ ), mix and heat for $5 \mathrm{~min}$ at $95^{\circ} \mathrm{C}$.

13) Load the samples in the precast gel.

14) Migrate for $15 \mathrm{~min}$ at $300 \mathrm{~V}$

15) Place the membrane and bottom stack on the cassette base, place the gel on top of the membrane, and roll the assembled sandwich to expel trapped air bubbles. Close and lock the cassette lid. Transfer for $3 \mathrm{~min}$ at $2.5 \mathrm{~V}$

16) Block the membrane with $5 \%$ of milk in PBS-Tween

17) Incubate membrane with 1:1000 diluted LC3B primary antibody/ $\beta$-Actin (C4) antibody in $5 \% \mathrm{w} / \mathrm{v}$ milk PBS-Tween at $4{ }^{\circ} \mathrm{C}$ with gentle shaking, overnight

18) Wash the membrane with PBS-Tween for $6 \times 5 \mathrm{~min}$

19) Incubate the membrane with horseradish peroxidase-conjugated secondary antibody for 
$1 \mathrm{~h}$ at room temperature

20) Wash the membrane with PBS-Tween for $6 \times 5 \mathrm{~min}$

21) Incubate the membrane with an ultrasensitive enhanced chemiluminescent substrate for 5

$\min$

11) Record images with a gel imaging system. Analyze and normalize the blot pictures with Image J software and using one-way ANOVA, Sidak's multiple comparisons test

12) See figure 3 for example of results obtained for RNVCs treated by 2 autophagy inducers (e.g. Digoxin and Digitoxigenin) allowing a comparison with the classical autophagy inducer Rapamycin. LC3II amounts have been normalized on $\beta$-actin for each treatment.

\subsection{Autophagosome formation using GFP-LC3 plasmid transfection}

\subsubsection{Equipment, materials and reagents}

\subsubsection{Materials and reagents}

1) GFP-LC3 plasmid is a gift from J.L. Perfettini (Inserm U1030, Gustave Roussy Institute, Villejuif, France)

2) Lipofectamine 2000 Transfection Reagent (Thermofisher, 11668030)

3) Opti-MEM ${ }^{\mathrm{TM}}$ I Reduced Serum Medium (Thermofisher, \#31985070)

4) Glass bottom culture dishes, NEST, \#80100

\subsubsection{Equipment}

1) Leica TCS SP8 STED confocal microscope 
2) Image J software (NIH, open source)

\subsubsection{Protocol}

1) Seed $0.4 \times 10^{6} \mathrm{RNVC}$ into $35 \mathrm{~mm}$, laminin-coated culture dishes $(10 \mu \mathrm{g} / \mathrm{ml})$ in $2 \mathrm{ml}$ of plating medium for overnight.

2) Change for fresh culture medium to remove dead cells

3) Prepare two $1.5 \mathrm{ml}$ falcon tubes, add $400 \mu \mathrm{l}$ Opti-medium into each one. First tube

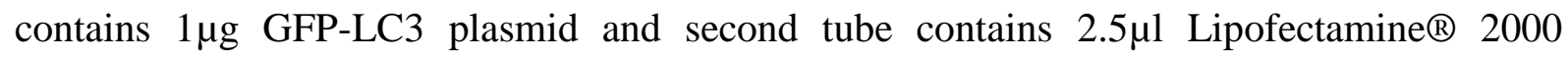
transfection reagent.

4) Add plasmid and Lipofectamine (total volume $800 \mu \mathrm{l}$ ) into the cell dishes.

5) $42 \mathrm{~h}$ after the transfection, treat the cells with $0.01 \%$ DMSO, $1 \mu \mathrm{M}$ Digitoxigenin, $1 \mu \mathrm{M}$ Digoxin and $3 \mu \mathrm{M}$ rapamycin in fresh Opti-MEM ${ }^{\mathrm{TM}}$ I Reduced Serum Medium for $6 \mathrm{~h}$.

6) After $6 \mathrm{~h}$, culture medium was replaced by $1 \mathrm{ml} 2.5 \mu \mathrm{g} / \mathrm{ml}$ Hoechst/PBS solution. Incubate it for $15 \mathrm{~min}$ at $37^{\circ} \mathrm{C}$ in a $5 \% \mathrm{CO}_{2}$ incubator

7) After $15 \mathrm{~min}$, remove the staining solution

8) Wash the cells for 3 times in warm PBS

9) Place each dish on Leica confocal microscope, select green channel and DAPI channel to detect green fluorescence and nucleus 


\section{Mitochondrial network analysis}

\subsection{Equipment, materials and reagents}

\subsubsection{Materials and reagents}

1) MitoTracker ${ }^{\mathrm{TM}}$ Red 580 (Thermofisher, M22425).

2) Calcein, AM, cell-permeant dye, (Thermofisher, C1430).

3) Glass bottom culture dishes, NEST, \#801002.

\subsubsection{Equipment}

1) Leica TCS SP8 STED confocal microscope

2) IMARIS software (Bitplane Company, Zurich, Switzerland)

\subsection{Experimental setup}

1) Seed $0.4 \times 10^{6} \mathrm{RNVC}$ into $35 \mathrm{~mm}$, laminin-coated culture dishes $(10 \mu \mathrm{g} / \mathrm{ml})$ in $2 \mathrm{ml}$ of plating medium overnight

2) Change culture medium to remove dead cells

3) Treat cells with $1 \mu \mathrm{M}$ Digitoxigenin, $1 \mu \mathrm{M}$ Digoxin, $3 \mu \mathrm{M}$ rapamycin (positive control), $0.01 \%$ DMSO (negative control) in serum free culture medium for $6 \mathrm{~h}$

4) After treatments, incubate cells with $200 \mathrm{nM}$ MitoTracker/PBS solution for $20 \mathrm{~min}$ at $37^{\circ} \mathrm{C}$

5) Remove MitoTracker solution and incubate cells with $4 \mu \mathrm{M}$ Calcein AM/PBS for 10min at $37^{\circ} \mathrm{C}$

6) Place each dish on Leica confocal microscope, select red channel and green channel to detect MitoTracker labeling of mitochondrial network and Calcein AM cell volume labeling 
respectively

7) Use $63 \mathrm{X}$ oil objective to obtain higher resolution images

8) Select the correct number of Z-frames to be scanned for 3D model reconstruction

9) Store data stacks as ***.lif with Leica confocal microscope

10) Open the data file with Imaris software, which processed and visualized in 3D

11) Before visualizing the volumes in high resolution in $3 \mathrm{D}$, crop the images to fit the scanned image or area of interest

12) Set the diameters and circularity of the individual mitochondria until most mitochondria are segmented.

13) Repeat the above steps for cell volume analysis

14) Select "analyze" to generate the report of mitochondria number and volume

15) Analyze the data using one-way ANOVA, Sidak's multiple comparisons test for cell volume and number of mitochondria as shown in Figure 5.

16) Please refer to note 3.

\section{Real time bioenergetic profile analysis}

\subsection{Equipment, materials and reagents}

\subsubsection{Materials and reagents}

1) H9c2(2-1) cardiomyocytes (ATCC® CRL-1446 ${ }^{\mathrm{TM}}$ ), Note 4.

2) Seahorse XFe96 FluxPak containing 18 XFe96 sensor cartridges, 20 XF96 cell culture microplates, and 1 bottle of Seahorse XF Calibrant Solution 500mL (Agilent, 102416-100) 
3) Seahorse XF base medium, sterile, 1 L, 2/pk, 102353-100

4) Oligomycin from Streptomyces diastatochromogenes, Sigma-Aldrich, O4876-5MG

5) 2-Deoxy-D-glucose (DG), Sigma-Aldrich, D8375

6) Carbonyl cyanide 4-(trifluoromethoxy)phenylhydrazone, FCCP, Sigma-Aldrich, C2920

7) Rotenone, Sigma-Aldrich, R8875-5G

8) Antimycin A, Streptomyces sp, Sigma-Aldrich, A8674

9) XF Cell Mito Stress Test Assay Medium: 100ml Seahorse XF base medium, sterile, containing $1 \mathrm{mM}$ pyruvate, $2 \mathrm{mM}$ glutamine, and $10 \mathrm{mM}$ glucose

10) XF Glycolysis Stress Test Assay Medium: 100ml Seahorse XF base medium, sterile, containing $2 \mathrm{mM}$ glutamine

\subsubsection{Equipment}

1) Seahorse $\mathrm{XFe} 96$ Analyzer and its analysis software Wave, Agilent

2) Multipette ${ }^{\circledR}$ E3, single-channel, with charging cable and Combitips advanced® assortment pack (1 Combitips ${ }^{\circledR}$ of each size) $1 \mu 1-50 \mathrm{ml}$, Eppendorf)

\subsection{Experimental setup}

\subsubsection{XF Cell Mito Stress Test}

1) One night before the assay, seed $\mathrm{H} 9 \mathrm{c} 2$ cells at 20,000 per well in 96 well plate. Turn on the Seahorse XFe96 Analyzer and allow temperature to stabilize at room temperature. Hydrate a sensor cartridge with $200 \mu \mathrm{l} \mathrm{XF}$ calibrant solution per well at $37{ }^{\circ} \mathrm{C}$ in a non- $\mathrm{CO}_{2}$ incubator overnight

2) On the day of the assay, treat cells by Digitoxigenin and Digoxin at $1 \mu \mathrm{M}, 3 \mu \mathrm{M}$ 
rapamycin (positive control), $0.01 \%$ DMSO (negative control) for $6 \mathrm{~h}$

3) Warm the pre-made XF Cell Mito Stress Test Assay Medium to $37^{\circ} \mathrm{C}$ and adjust pH to 7.4 \pm 0.1 at $37^{\circ} \mathrm{C}$

4) Retrieve the cell plate from the $\mathrm{CO}_{2}$ incubator. Note the time

5) Check the cells under the microscope to confirm cell viability, morphology, seeding uniformity and absence of contamination and make sure no cells were plated in the background correction wells

6) Wash cells with XF Cell Mito Stress Test Assay Medium for 3 times. Remove all but 20 $\mu l$ of the culture medium from each well. Rinse cells two times with $200 \mu$ of assay medium. Add assay medium to each well for a final volume of $180 \mu \mathrm{l} /$ well

7) Place the plate in a $37^{\circ} \mathrm{C}$ incubator without $\mathrm{CO}_{2}$ for one hour prior to the assay

8) Prepare the stock solution (10X) of each injection with XF Cell Mito Stress Test Assay Medium, $20 \mu \mathrm{M}$ oligomycin, $10 \mu \mathrm{M}$ FCCP and $5 \mu \mathrm{M}$ Rotenone and Antimycin A

9) Get a hydrated cartridge from the non- $\mathrm{CO}_{2}$ incubator. Load the cartridge in each port as outlined below

a. Port $\mathrm{A}-2 \mu \mathrm{M}$ oligomycin final concentration in the well. Load $20 \mu \mathrm{l}$ of your 10x stock into each Port A.

b. Port B $-1 \mu \mathrm{M}$ FCCP final concentration in the well. Load $22 \mu 1$ of your 10x stock into each Port B.

c. Port $\mathrm{C}-0.5 \mu \mathrm{M}$ Rot/AA final concentration in the well. Load $25 \mu \mathrm{l}$ of your 10x stock into each Port C.

10) Create or load your assay template on the XF Controller. Default Mix-Wait-Measure 
times are $3 \min -0 \min -3 \min$. Usually 3 basal rate measurements are taken prior to the first injection; then 3 rate measurements after each injection

11) On the Run Screen, Press Start and load the cartridge

12) When prompted by the software, replace the Utility Plate with the Cell plate. Press Continue

13) After the assay finished, open the Seahorse data by using WAVE software

14) Export the data to XF Cell Mito Stress Test Assay Generator and Analyze the data using one-way ANOVA, Sidak's multiple comparisons test

14) Please refer to note 5 and 6.

\subsubsection{XF Glycolysis Stress Test}

1) One night before the assay, seed $20,000 \mathrm{H} 9 \mathrm{c} 2$ cells per well in 96 well plate. Turn on the Seahorse XFe96 Analyzer and allow temperature to stabilize at room temperature. Hydrate a sensor cartridge with $200 \mu \mathrm{XF}$ calibrant solution per well at $37{ }^{\circ} \mathrm{C}$ in a non- $\mathrm{CO}_{2}$ incubator overnight

2) On the day of the assay, treat cells by Digitoxigenin and Digoxin at $1 \mu \mathrm{M}, 3 \mu \mathrm{M}$ rapamycin (positive control), $0.01 \%$ DMSO (negative control) for $6 \mathrm{~h}$

3) Warm the pre-made XF Glycolysis Stress Test Assay Medium to $37^{\circ} \mathrm{C}$. Adjust pH to $7.4 \pm$ 0.1 at $37^{\circ} \mathrm{C}$

4) Retrieve the cell plate from the $\mathrm{CO}_{2}$ incubator. Note the time

5) Check the cells under the microscope to confirm cell health, morphology, seeding uniformity and absence of contamination and make sure no cells were plated in the background correction wells 
6) Wash cells with XF Glycolysis Stress Test Assay Medium for 3 times. Remove all but 20 $\mu l$ of the culture medium from each well. Rinse cells two times with $200 \mu$ l of assay medium. Add assay medium to each well for a final volume of $180 \mu \mathrm{l} /$ well

7) Place the plate in a $37^{\circ} \mathrm{C}$ incubator (without $\mathrm{CO}_{2 \text { ) }}$ for one hour prior to the assay

8) Prepare the stock solution (10X) of each injection with XF Glycolysis Stress Test Assay Medium, 100mM glucose, $20 \mu \mathrm{M}$ oligomycin and $500 \mathrm{mM}$ 2-DG

9) Get a hydrated cartridge from the non- $\mathrm{CO}_{2}$ incubator. Load the cartridge in each port as outlined below

a. Port A $-10 \mathrm{mM}$ glucose final concentration in the well. Load $20 \mu \mathrm{l}$ of your 10x stock into each Port A.

b. Port $\mathrm{B}-2 \mu \mathrm{M}$ oligomycin final concentration in the well. Load $22 \mu \mathrm{l}$ of your 10x stock into each Port B.

c. Port C $-50 \mathrm{mM} 2-\mathrm{DG}$ final concentration in the well. Load $25 \mu \mathrm{l}$ of your 10x stock into each Port C.

10) Create or load your assay template on the XF Controller. Default Mix-Wait-Measure times are $3 \min -0 \min -3 \min$. Usually 3 basal rate measurements are taken prior to the first injection; then 3 rate measurements after each injection

11) On the Run Screen, Press Start and load the cartridge

12) When prompted by the software, replace the Utility Plate with the Cell plate. Press Continue

13) After the assay finished, open the Seahorse data by using WAVA software

14) Export the data to XF Glycolysis Stress Test Assay Generator and analyze the data using 
one-way ANOVA, Sidak's multiple comparisons test

15) Please refer to note 5 and 6. 


\begin{abstract}
In the perspective to evaluate the toxicity of drug candidates or the exploration of intracellular signalling pathways of cell stress response and pathophysiological conditions, we propose to evaluate cell death, autophagy, mitochondrial network and energetic metabolism by a series of optimized joint protocols for neonatal primary rat cardiomyocytes or $\mathrm{H} 9 \mathrm{c} 2$ cardiac cell line in 96 well microtiter plates. We used Digitoxigenin and Digoxin, two cardiac glycosides, and Rapamycin as control drugs, for inhibition of oxidative stress-induced cell death and autophagy induction respectively.
\end{abstract}




\section{Acknowledgments}

This work has been funded by Institut National de la Santé et de la Recherche Médicale-INSERM, the Investment for the Future program ANR-11-IDEX-0003-01 within the LABEX ANR-10-LABX-0033, INCa N²017-168. Catherine Brenner is Research Director at Centre National de la Recherche Scientifique - CNRS, Dawei LIU was supported by a fellowship from the China Scholarship Council. 


\section{References}

1. Findlay, S.G., et al., Chronic cardiovascular toxicity in the older oncology patient population. J Geriatr Oncol, 2019. 10(5): p. 685-689.

2. Mancilla, T.R., B. Iskra, and G.J. Aune, Doxorubicin-Induced Cardiomyopathy in Children. Compr Physiol, 2019. 9(3): p. 905-931.

3. Kroemer, G., L. Galluzzi, and C. Brenner, Mitochondrial membrane permeabikisation in cell death? Physiol. Rev. 87 (1):p 99-138.

4. Korzeniewski, C. and D.M. Callewaert, An enzyme-release assay for natural cytotoxicity. J Immunol Methods, 1983. 64(3): p. 313-20.

5. Nachlas, M.M., et al., The determination of lactic dehydrogenase with a tetrazolium salt. Anal Biochem, 1960. 1: p. 317-26.

6. Levine, B., N. Mizushima, and H.W. Virgin, Autophagy in immunity and inflammation. Nature, 2011. 469(7330): p. 323-335.

7. Kabeya, Y., et al., LC3, a mammalian homologue of yeast Apg8p, is localized in autophagosome membranes after processing. Embo j, 2000. 19(21): p. 5720-8.

8. Martinou, J.C., Apoptosis. Key to the mitochondrial gate. Nature, 1999. 399(6735): p. 411-2.

9. Brenner, C. and G. Kroemer, Apoptosis. Mitochondria--the death signal integrators. Science, 2000. 289(5482): p. 1150-1.

10. Pervaiz, S., et al., Redox signaling in the pathogenesis of human disease and the regulatory role of autophagy. Int Rev Cell Mol Biol, 2020. 352: p. 189-214.

11. Green, D.R., L. Galluzzi, and G. Kroemer, Cell biology. Metabolic control of cell death. Science, 2014. 345(6203): p. 1250256.

12. Wang, Z., et al., A cardiac mitochondrial cAMP signaling pathway regulates calcium accumulation, permeability transition and cell death. Cell Death Dis, 2016. 7: p. e2198.

13. Klionsky, D.J., et al., Guidelines for the use and interpretation of assays for monitoring autophagy (3rd edition). Autophagy, 2016. 12(1): p. 1-222.

14. Liu, D., et al., PDE2 regulates membrane potential, respiration and permeability transition of rodent subsarcolemmal cardiac mitochondria. Mitochondrion, 2019. 47: p. 64-75.

15. Chen, M.H., R. Kerkelä, and T. Force, Mechanisms of cardiac dysfunction associated with tyrosine kinase inhibitor cancer therapeutics. Circulation, 2008. 118(1): p. 84-95.

16. Carvalho, F.S., et al., Doxorubicin-induced cardiotoxicity: from bioenergetic failure and cell death to cardiomyopathy. Medicinal research reviews, 2014. 34(1): p. 106-135. 


\section{Notes}

Note 1. If not indicated, reagents or kits are from SIGMA. When needed for reproducibility, references of some compounds or materials have been indicated and then, suppliers and catalog numbers are indicated within bracket.

Note 2. LDH assay can be combined with another assay using the treated cells such as PI assay. In addition, $\mathrm{LDH}$ assay can be perform sequentially after cell treatments or on $-20^{\circ} \mathrm{C}$ frozen cell supernatants, whereas PI staining has to be performed immediately after cell treatments. Due to its intrinsic fluorescence, PI could induce some interference with other fluorescent probes with similar spectral properties.

Note 3. An increase in the number of mitochondria can be related to fission events if the mitochondrial network fluorescence is similar between a control condition and a treatment. If the number of mitochondria increase concomitantly with an increase of mitochondrial network fluorescence, this event is related to mitochondrial biogenesis.

Note 4. H9c2 cells have been used instead of RNVC, because, in our hands, their ability to attach and remain viable during the time course of experiment was better.

Note 5. It is mandatory to check the cell treatment affects the cell number during the experiment. And, if yes, it is necessary to normalize the OCR and ECAR results. This can be done, for example, by determining the amount of protein/well and expressing results in $\mathrm{pmol} / \mathrm{min} / \mathrm{mg}$ with the use of the wave software.

Note 6. Many graphs with other parameters can be drawn using the Wave Agilent software. 


\section{Figure Legend}

Figure 1. Protective effects of Digitoxigenin and Digoxin from $\mathrm{H}_{2} \mathrm{O}_{2}$-induced necrosis in neonatal rat ventricular cardiomyocytes (RNVC) measured by LDH assay. Six hour-pre-treatment of RNVC by $10 \mu \mathrm{M}$ Digitoxigenin and $10 \mu \mathrm{M}$ Digoxin prove to efficiently inhibit necrosis induced by $300 \mu \mathrm{M} \mathrm{H}_{2} \mathrm{O}_{2}$. Each experiment has been reproduced at least three times. Data are presented as mean \pm standard error to the mean (SEM) with one-way ANOVA, Sidak's multiple comparisons test. ****, $\mathrm{p}<0.0001$ treatment vs $300 \mu \mathrm{M} \mathrm{H}_{2} \mathrm{O}_{2}$.

Figure 2. Protective effects of Digitoxigenin and Digoxin from $\mathrm{H}_{2} \mathrm{O}_{2}$-induced necrosis in RNVCs using PI staining. RNVCs membrane permeabilisation was measured with propidium iodide. Data are presented as mean \pm SEM with one-way ANOVA, Sidak's multiple comparisons test. $* * *, \mathrm{p}<0.001$ treatment $v$ s DMSO.

Figure 3. Quantification of LC3- II amount. Protein amount of LC3- II was analyzed by western blot in RNVCs treated by $0.01 \%$ DMSO, $1 \mu \mathrm{M}$ Digitoxigenin, $1 \mu \mathrm{M}$ Digoxin and $3 \mu \mathrm{M}$ rapamycin for $6 \mathrm{~h}$ or untreated (Co.). Data have been normalized on $\beta$-actin amount and are presented as mean \pm SEM with one-way ANOVA, Sidak's multiple comparisons test. *, $\mathrm{p}<$ 0.05 treatment $v s$ DMSO.

Figure 4. Quantification of GFP-LC3 dots. RNVCs were transfected with a GFP-LC3 plasmid to monitor the autophagosome formation. Following GFP-LC3 plasmid transfection, GFP-LC3 green fluorescent dots corresponding to autophagosomes were visualized by fluorescence microscopy and quantified by Image $\mathrm{J}$ to monitor autophagosome formation. Co. (untreated). Data are presented as mean \pm SEM with one-way ANOVA, Sidak's multiple comparisons test. $* * *, \mathrm{p}<0.001$ vs $0.01 \%$ DMSO.

Figure 5. Effects of Digitoxigenin and Digoxin on mitochondrial network and cell volume. RNVCs were treated by $1 \mu \mathrm{M}$ Digitoxigenin and Digoxin for $6 \mathrm{~h}$ and the mitochondrial network 
was labelled by $200 \mathrm{nM}$ Mitotracker Red (A, B) and the cell volume by calcein (A, C). Mitochondrial network and individual mitochondria were then analyzed by using the Leica confocal microscope and IMARIS software. Data are presented as mean \pm SEM with one-way ANOVA, Sidak's multiple comparisons test. *, $\mathrm{p}<0.05,{ }^{*}, \mathrm{p}<0.01, * * *, \mathrm{p}<0.001$ vs DMSO.

Figure 6. Effects of Digitoxigenin and Digoxin on mitochondrial oxygen consumption rates. Mitochondrial respiration functions (Basal respiration (A) and spare respiratory capacity (B)) of H9c2 cells were measured by mito stress test on the XFe96 Extracellular Flux Analyzer. OCR (oxygen consumption rate). OCR data presented are presented as mean \pm SEM with one-way ANOVA, Sidak's multiple comparisons test. **, $\mathrm{p}<0.01$, ***, $\mathrm{p}<0.001$ vs DMSO.

Figure 7. Glycolysis stimulating effects of Digitoxigenin and Digoxin. Glycolytic functions (Glycolysis (A) and Glycolytic capacity (B)) of H9c2 cells were measured by glycolytic stress test on the XFe96 Extracellular Flux Analyzer, ECAR (Extracellular acidification rate). Results have been normalized/well. ECAR data are presented as mean \pm SEM with one-way ANOVA, Sidak's multiple comparisons test. ${ }^{*}, \mathrm{p}<0.05 ; * * *, \mathrm{p}<0.001$ vs DMSO. 